Cinémas

Revue d'études cinématographiques

Journal of Film Studies

\title{
Le Cinéma holographique : les expérimentations de Claudine Eizykman et Guy Fihman
}

\section{René Prédal, Claudine Eizykman et Guy Fihman}

Volume 1, numéro 3, printemps 1991

Nouvelles technologies : nouveaux cinémas?

URI : https://id.erudit.org/iderudit/1001066ar

DOI : https://doi.org/10.7202/1001066ar

Aller au sommaire du numéro

Éditeur(s)

Cinémas

ISSN

1181-6945 (imprimé)

1705-6500 (numérique)

Découvrir la revue

Citer cet article

Prédal, R., Eizykman, C. \& Fihman, G. (1991). Le Cinéma holographique : les expérimentations de Claudine Eizykman et Guy Fihman. Cinémas, 1(3), 61-76. https://doi.org/10.7202/1001066ar
Résumé de l'article

Un texte à vocation informative présente l'oeuvre des « inventeurs » français du cinéma holographique, selon une articulation qui développe la problématique de l'expérimentation artistique versus l'expérimentation technologique, la dimension volumétrique et le concept de " sculptures en mouvement ". Suit un texte à caractère réflexif des cinéholographes, relatif à l'intérêt de la cinéholographie pour la conquête de la troisième dimension et la primauté accordée à la vision sur le monde vu. 


\title{
Le Cinéma holographique: les expérimentations de Claudine Eizykman et Guy Fihman
}

\section{René Prédal, Claudine Eizykman et Guy Fihman}

\begin{abstract}
RÉSUMÉ
Un texte à vocation informative présente l'œuvre des «inventeurs» français du cinéma holographique, selon une articulation qui développe la problématique de l'expérimentation artistique versus l'expérimentation technologique, la dimension volumétrique et le concept de «sculptures en mouvement». Suit un texte à caractère réflexif des cinéholographes, relatif à l'intérêt de la cinéholographie pour la conquête de la troisième dimension et la primauté accordée à la vision sur le monde vu.
\end{abstract}

\begin{abstract}
The work of the French "Inventors" of holographic cinema is presented in the course of a discussion of artistic versus technical experimentation, the volumetric dimension and the concept of "moving sculpture". This is followed by a text in which the cineholographers reflect on cineholography, seen as an attempt to conquer the third dimension and as a medium which accords primacy to the act of seeing rather than to the world seen.
\end{abstract}




\section{Claudine Eizykman et Guy Fihman: de la cinéhologra- phie au cinéma holographique}

\section{René Prédal}

«Inventeurs» français du cinéma holographique, Claudine Eizykman et Guy Fihman nous paraissent moins soucieux d'apporter le relief au cinéma (ce qui, en somme, s'était déjà fait), que de s'attacher à doter de mouvement l'hologramme, ce qui constitue une tout autre démarche et fonde littéralement une forme de représentation nouvelle par rapport au septième art. Il est vrai que, «étant données les différences technologiques, esthétiques et visuelles entre le cinéma stéréoscopique et le cinéma holographique, celui-ci représente aussi bien la concrétisation du cinéma en relief, qui reste partiel en stéréoscopie, que celle de l'holographie en mouvement» $(\mathrm{Cl} \text {. E. et G. F. })^{1}$.

\section{Expérimentation artistique - expérimentation techno- logique}

Si la première manifestation de la cinéholographie a lieu en 1982, Claudine Eizykman et Guy Fihman ne sont pas à ce moment des nouveaux venus sur le front de l'expérimentation dans les arts cinégraphiques. Dès le début des années 70 , en effet, ils réalisent - déjà ensemble ou séparément - des films de métrages divers alors classés dans le domaine de l'expérimental indépendant:

Les notions de court ou de long métrage n'ont heureusement pas cours dans le cinéma expérimental, où la longueur ou la durée d'un film est déterminée par le cinéaste en fonction de sa logique artistique et non en raison de catégories économiques ou réglementaires. Le fait que des films comme Trois couches suffisent (G.F., 52 minutes), Lapse (C.E., 80 minutes) ou Bruine Sqamma (C.E., 120 minutes) soient généralement répertoriés comme des "courts métrages» pose la question de savoir si cette opinion relève d'une intention dévalorisante ou d'une appréciation flatteuse!» (Cl. E. et G.F.).

À ce titre de cinéastes expérimentaux, ils donnent chacun un texte au fameux numéro spécial de la Revue d'esthétique coordonné par Dominique Noguez, Cinéma, théorie, lectures.

Pour Alain Sudre, les propositions filmiques de G. Fihman partent de l'idée que le changement de couleur d'un même objet est cinématographiquement équivalent à la synthèse du mouvement. Ultra-rouge-infra-violet (1974) et Trois couches suffisent 
(1978) examinent systématiquement les possibilités esthétiques de ce postulat. Utilisant la trichromie du support, le cinéaste s'éloigne délibérément du spectre des «couleurs naturelles» dans des compositions qui s'organisent comme une «suite de tableaux». Quant à $\mathrm{Cl}$. Eizykman, son œuvre s'inscrirait «dans le prolongement des films de Peter Kubelka et de Paul Sharits dont les propositions adoptaient le photogramme comme unité de travail. Avec le temps, le procédé de montage de grappes d'images s'enrichira d'incrustations, de solarisations et de superpositions» (Sudre). Ces appréciations ne sont évidemment données qu'à titre purement indicatif et, pour les auteurs,

(...) il serait vain de vouloir rectifier dans une simple remarque tout ce qu'a de partiel et de partial une notule de dictionnaire ou une notice de critique. Invitons le lecteur à prendre directement connaissance des films et des textes (Cl. E. et G. F.).

Dans le même temps, Claudine Eizykman prépare une thèse de philosophie (doctorat soutenu en 1974), travaille au Service de la recherche de l'ORTF, puis s'engage dans les études du Laboratoire d'expérimentations dans les arts cinégraphiques (LEAC). En tant qu'enseignante à l'Université de Paris VIII, elle participe avec Guy Fihman à l'ouvrage Du cinéma selon Vincennes. Son livre La Jouissance-cinéma sort en plein bouillonnement idéologique post-soixante-huitard et suscite quelques débats passionnés ${ }^{2}$ dans les cénacles de théoriciens, sa manière de désigner le cinéma dominant sous le sigle N.R.I. (forme narrative-représentative-industrielle) connaissant un certain succès à la fin de la décennie. Avec le recul, Jacques Kermabon considère en 1988 que l'étude constitue un essai original, quoiqu'un peu daté, qui veut «penser le tout du cinéma en inscrivant les dimensions psychologique, économique, sociologique, sémantique, etc..., à chaque fois partielles, dans une problématique pensée en termes de circulations de flux énergétiques inspirée de JeanFrançois Lyotard» (p. 192). Sans doute serait-il "plus approprié de dire que LaJouissance-cinéma procède à l'établissement du réglage du Cinéma N.R.I., qui occupe une tranche du spectre filmique, selon une triple détermination corrélée: psychique, esthétique et sociale» (Cl. E. et G.F.).

En 1976, elle fonde en outre la revue Melba qu'elle dirige jusqu'en 1979: «Il faut savoir arrêter la publication d'une revue, surtout lorsqu'on estime que certains des buts que l'on s'était fixés ont été atteints» (Cl. E. et G. F.). Ses initiatives multiformes balisent en somme tout le champ ENTRE théorie et pratique au lieu de se borner à additionner les deux formes d'activités créatives et réflexives. Par là, elle appartient aux 
«mutants-cinéastes pour lesquels la gestion d'un journal, d'une coopérative, la fabrication filmique et la fabrication d'un journal sont autant de plis de cette mutation» (Eizykman, p. 234).

\section{La dimension volumétrique}

Avec Claudine Eizykman et Guy Fihman, le cinéma holographique connaît dans les années 80 , à la fois son temps des magiciens, des artistes et des techniciens, à partir de comportements que l'on pourrait qualifier de «philosophiques» au sens ancien du terme, c'est-à-dire s'attachant à une connaissance raisonnée englobant aussi bien les sciences de la nature que les sciences humaines. Ceci dit, ils n'ont ni l'un ni l'autre une formation scientifique (ils ne sont pas licenciés en physique), mais sont devenus spécialistes des domaines de l'optique et de la perception où il leur arrive d'en remontrer aux hommes de laboratoire qui ne parviennent pas toujours à mettre leurs recherches en perspective et ne saisissent pas tous les enjeux de leurs découvertes. $\mathrm{Cl}$. Eizykman et G. Fihman se passionnent quant à eux pour l'épistémologie, établissant un rapport critique et réflexif avec les sciences.

Alors que le cinéma s'épuise outre-Atlantique à poursuivre l'impression de RELIEF (système stéréoscopique avec lunette bleu-rouge), les deux chercheurs français veulent obtenir plutôt un effet de VOLUME, en partant de l'hologramme dont enregistrement et lecture se font au laser sur le même support. On peut alors bouger un peu pour apprécier pleinement ce volume sous divers angles. Les premiers essais dans cette direction remontent à la fin des années 70, mais ce sont des expériences de laboratoire sans aucune dimension de spectacle. Seul le professeur Komar à Moscou présente en 1976 un «cinéma théâtral holographique» mais qui fatigue considérablement la vue.

En $1982^{3}$, à la fondation Hugot du Collège de France, Claudine Eizykman et Guy Fihman choisissent le 22 avril, date du centenaire du fusil photographique de E.J. Marey, en vue de monter le premier spectacle holographique, intitulé Vols d'oiseaux et élaboré au laboratoire d'optique de Besançon affilié au CNRS. Enregistré sur plaque de verre, c'est une sorte d'animation, puisque l'objet holographié n'est pas un véritable oiseau en vol, mais la série des figurines de goélands en bronze de Marey conservées au Collège de France. En fait,

(...) la date du 22 avril 1982 n'a pas été choisie pour «monter» mais pour commencer à «montrer» le spectacle des Vols d'oiseaux en hommage à Étienne-Jules Marey; il s'agissait d'une animation entièrement holographique, c'est-à-dire d'une synthèse de mouvement réalisée à partir de l'analyse du vol du goéland que consti- 
tuent les sculptures de Marey: ces «figurines en relief» doivent être considérées comme la première forme de cinéma en relief intégral (Cl. E. et G.F.).

La plaque écran fait $50 \mathrm{~cm}$ sur 60 . Vingt images ont été enregistrées sur ce même support alors que les expérimentateurs antérieurs n'avaient pu atteindre que sept positions différentes au maximum; le saut quantitatif est donc important. Dans les excellentes conditions de vision de l'époque, sept ou huit spectateurs peuvent voir le goéland avancer quelques secondes du fond de l'image vers l'avant, semble-t-il à partir de plusieurs mètres de loin jusqu'à quelques centimètres de près. Malheureusement, nous n'avons pu assister aux présentations privées et publiques de 1982, dans lesquelles les cinéhologrammes étaient restitués en bichromie au moyen de deux lasers. Notre impression personnelle est donc sans doute moins forte que celle des premiers spectateurs car nous n'avons pu voir en 1990 qu'une

\begin{abstract}
(...) variante aux performances sensiblement plus réduites, en format $(30 \times 40 \mathrm{~cm}$ au lieu de $50 \times 60 \mathrm{~cm})$, en nombre de vues $(10$ au lieu de 20), en sujets (un seul oiseau au lieu de dix) et en volume scénique (un vol stationnaire au lieu de vols circulaires ou dans la profondeur de plusieurs mètres) et une restitution en lumière incohérente au moyen d'un séquenceur de lumières blanches (Cl. E. et G.F.).
\end{abstract}

En 1985 au CESTA (Centre d'étude des systèmes et des technologies avancées), il s'agit d'un prototype fabriqué à partir d'un appareil pour prises de vues aériennes qui utilise une pellicule géante de $126 \mathrm{~mm}$ sans perforations et se déplaçant en continu (ce sont les pulsions du laser qui individualisent chaque prise et non le jeu de l'obturateur):

Aussi bien la caméra $126 \mathrm{~mm}$ que la Visu-laser 126, comme
d'ailleurs les matériels précédents et ultérieurs, ont été et sont réa-
lisés de manière indépendante et sous notre pleine responsabilité.
Cependant, nous avons demandé à des ingénieurs de
l'Aérospatiale de tester différentes caractéristiques du laser pour
l'application de prises de vues cinéholographiques. Mais ni ces
ingénieurs très compétents ni l'Aérospatiale ne sont pour rien dans
l'élaboration de notre équipement spécifique. Insinuer le contraire
vérifierait l'adage selon lequel «on ne prête qu'aux riches", faisant
en outre apparaître le présupposé selon lequel seuls les techniciens
peuvent concevoir des machines (Cl. E. et G.F.).

Un $n u$ se visionne encore à travers une petite fenêtre, mais cette fois avec les deux yeux. Malheureusement, la vision n'atteint pas la netteté de Vols d'oiseaux et les imperfections techniques (un laser bas de gamme pas assez puissant, une coloration verdâtre pas très heureuse) sont durement ressenties par 


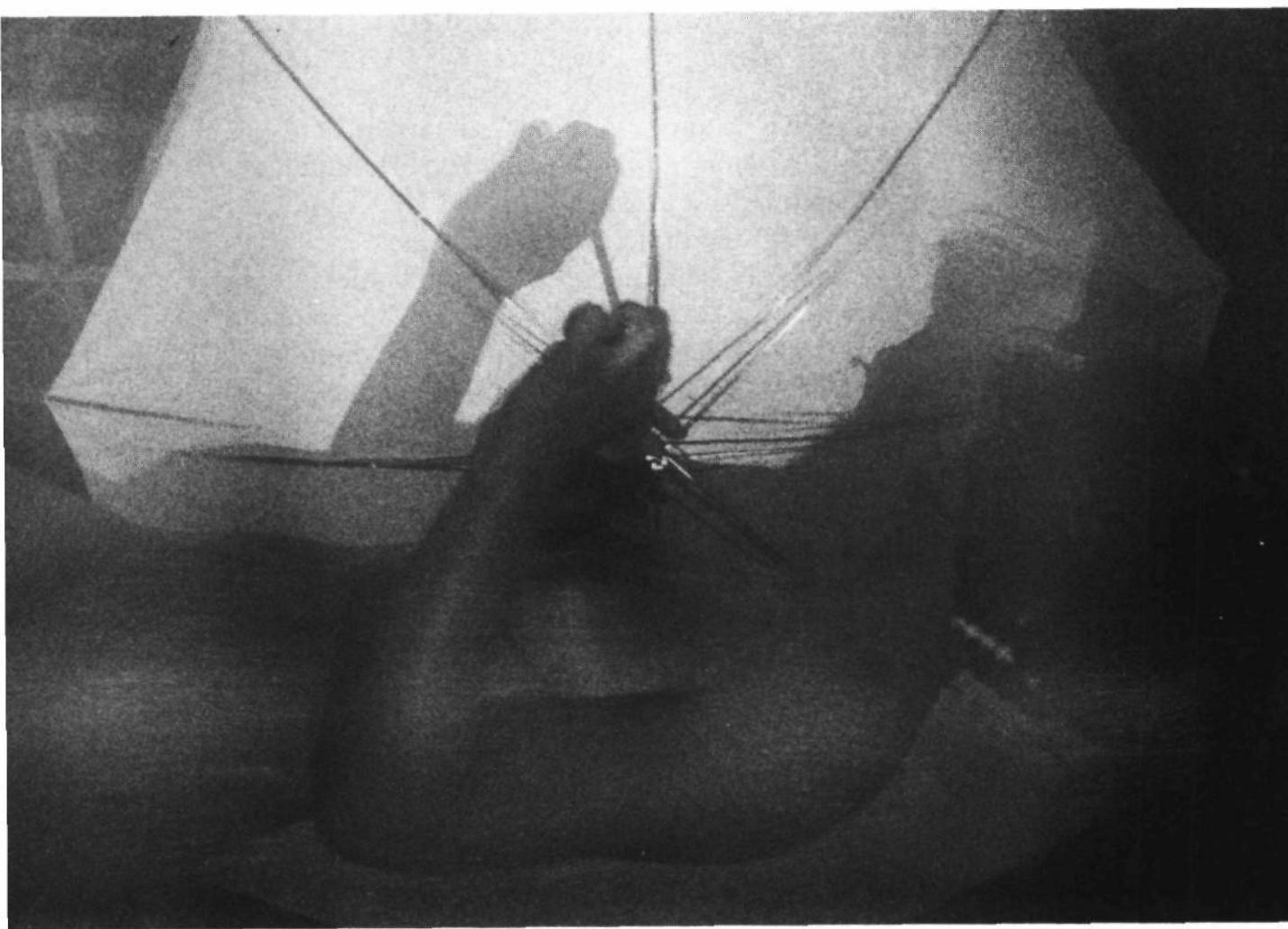

Un $n u$, holo-film en $126 \mathrm{~mm}$, de Claudine Eizykman et Guy Fihman avec le concours de Christian Le Floc'h et Jacques Bouteyre (1985)

l'observateur: il y a du moirage, peu de contraste, pas assez de luminosité, des stries alternativement claires et foncées qui résultent des conditions dans lesquelles a été réalisé $U n n u$, «à savoir celles du test d'un laser conçu pour une application scientifique, en vue de son utilisation en cinéholographie. Certaines des caractéristiques des lasers Yag de cette époque (1985!), aujourd'hui dépassés, entraînaient diverses imperfections, et nous avons choisi de ne pas les éliminer» (Cl. E. et G.F.). On voit en tous cas nettement une momie qui se déroule pour dégager une femme nue ${ }^{4}$; l'impression de volume est troublante et cette minute et demie provoque comme un appel de fiction qui donne à l'événement une coloration beaucoup plus intense que celle pouvant émaner d'une simple curiosité scientifique.

Les travaux se poursuivent actuellement, mais aucune autre présentation n'a été faite depuis cinq ans par les chercheurs: 
l'étape suivante consistera-t-elle à projeter l'holofilm au lieu de devoir l'observer directement sur le support? Rien n'est moins sûr, car chaque avancée nouvelle demande la construction du matériel nécessaire à l'expérimentation et les crédits des laboratoires universitaires ne sont jamais bien importants.

\section{Des «sculptures en mouvement»}

C'est la formule employée par Guy Fihman au carrefour «Convergence II: au-delà de la technologie», tenu à Montréal en 1986 où il opposait la cinéholographie au cinéma qui, lui, serait de la «peinture en mouvement». En fait, $\mathrm{Cl}$. Eizykman et G. Fihman sont en position d'INVENTEURS, parlant volontiers de Léonard de Vinci ou de Marey qui a dû fabriquer son fusil photographique comme eux-mêmes la chaîne complète de leur appareillage, avec le mélange de bricolage et de sophistication typique de ce genre de SAVANTS (mot qu'ils préfèrent à celui de SCIENTIFIQUES) toujours entre l'évier de leur cuisine et les installations de la Nasa.

Les sujets de leurs deux réalisations majeures (Vols d'oiseaux, Un nu) ne sont évidemment pas hasardeux, les sculptures de Marey renvoyant au cinéma des origines et le nu accusant la filiation des arts plastiques, ce qui transcende l'invention technique pour la replacer sur la ligne du devenir des activités humaines. Pour parler clair, ils n'ont pas cinéholographié n'importe quel barreau de chaise et choisissent aussi très soigneusement les moments de divulguer leurs découvertes qu'ils mettent en spectacle et en situation de façon presque symbolique.

Vols d'oiseaux m'a semblé travailler prioritairement la PROFONDEUR. La place idéale du spectateur est déterminée géométriquement, très près de l'écran, d'où il peut apprécier les diverses composantes d'un relief littéralement sculpté par le mouvement. Un nu s'attaque quant à lui au CADRE. Celui ressenti à la vision paraît beaucoup plus large que celui matérialisé par le cube de l'appareil: il «dépasse» des deux côtés, le contenant semble plus petit que le contenu. En mettant l'oeil à la fenêtre, on élargit son champ visuel au lieu de le restreindre.

Les particularités de l'image holographique - que Michel Larouche définit comme tridimensionnelle, transparente, immatérielle, en lévitation, diaphane, quasi irréelle - ne manquent donc pas de poser bien des problèmes aux théoriciens de la représentation. Ainsi, l'apparition d'une authentique troisième dimension ne se traduirait nullement par un gain de réalisme. Bien au contraire, l'espace à l'intérieur de l'appareil constitue un ailleurs étrange et fascinant aux limites logiquement indiscernables mais néanmoins ressenties: s'il n'y a plus de cadre, un hors- 
champ subsiste! L'unicité du support-écran (comme si au cinéma l'écran était la pellicule ou réciproquement) libère l'oeil humain en même temps qu'il le piège, d'autant plus qu'alors que le cinéma est un art reproductible, la cinéholographie en revient à l'œuvre unique, un peu comme un tableau qui - en outre - ne serait visible que par une poignée d'amateurs réunis dans des conditions tout à fait particulières.

Sans doute est-il d'ailleurs encore trop tôt pour se demander si l'on pourra élaborer un véritable langage cinéholographique. $\mathrm{Cl}$. Eizykman et G. Fihman viennent en effet tout juste de passer le cap d'une sorte de précinéma holographique et ce qu'ils nous ont fait voir conduit à penser que ce langage deviendra peut-être aussi différent de celui du cinéma que ce dernier l'est présentement du langage théâtral ou pictural. D'ailleurs

(...) la notion de précinéma holographique, reprise du découpage introduit par certains historiens du cinéma photographique, vise les formes premières de cinéma (photographique ou holographique), c'est-à-dire celles qui précèdent la phase industrielle. En fait, le "précinéma» est l'avant-Cinématographe, mais c'est déjà du cinéma ( $\mathrm{Cl}$. E. et G.F.).

Les deux inventeurs sont en tous cas très attentifs à juguler les dérives de sens liées à des emplois abusifs de termes mal maîtrisés. Toute affirmation les concernant est disséquée, retournée, passée au crible... et si nous disons qu'elle est même finalement à chaque fois rejetée, ils contestent également cette assertion:

(...) parce que d'abord il devrait s'agir de notre travail et non de nous. Mais surtout, il est certain que la problématique philosophique, technique et esthétique, qu'implique le cinéma holographique remet en question tout un savoir que l'on croyait acquis, qu'il nous a fallu désapprendre afin de pouvoir apprendre. Nous comprenons les difficultés que peuvent rencontrer nos interlocuteurs (d'autant plus qu'à l'occasion des diverses présentations, ils ont été nombreux et provenant d'horizons très différents), mais nous estimons préférable, en relevant telle erreur ou imprécision, de faire sentir cette mutation conceptuelle (Cl. E. et G. F.).

Leur recherche n'est donc nullement indéfinissable ni inexprimable, mais à la condition de l'envisager dans sa réelle complexité. Refusant d'être assimilés à des scientifiques, ils ont par contre toujours affirmé venir du cinéma, surtout si l'on veut bien ne pas méconnaître ses origines philosophiques. Aussi n'acceptent-ils de s'exprimer que sur ce qui est déjà passé, dévoilé, et préfèrent-ils garder secrètes les issues de leurs travaux en cours. Cherchant du nouveau, aucun vocabulaire ne serait en somme adéquat pour en rendre compte: la chose n'existant pas, le 
concept non plus et encore moins les mots pour le dire. On ne peut donc ni les contester radicalement, ni leur rendre totalement justice. Ils campent seuls en terra incognita. Tout rapprochement les met mal à l'aise. Aucun essai n'est comparable au leur, non du point de vue de la qualité des résultats mais, plus profondément, au niveau de l'essence même du projet.

Sans doute répondraient-ils aussi peu volontiers au questionnement américain direct (What is your statement?) qu'à la malice d'Abraham Moles aimant demander aux chercheurs universitaires qu'on lui présente: «Mais qu'avez-vous trouvé?». Ils préfèrent que l'amateur s'approche lentement, leurs révélations ne pouvant toucher vraiment que les initiés. Pourtant, sous la force de sollicitations pressantes, ils acceptent parfois de faire part de quelques réflexions sur leurs travaux, parvenant alors à une remarquable rigueur dans l'exposé5 dont le lecteur pourra juger lui-même par le texte suivant qui nous permet d'affirmer sans ambages: oui, le cinéma holographique existe, nous l'avons rencontré.

Université de Caen

\section{NOTES}

1 Les interventions de Claudine Eizykman et de Guy Fihman, signées Cl. E. et G. F. dans le cours de cet article, représentent les observations des «sujets» suscitées par la lecture de la première mouture de notre texte rédigé après notre rencontre en octobre 1990. De sa lecture attentive et annotée résulte ce jour le diptyque ainsi proposé aux lecteurs de Cinémas : d'une part notre propre présentation à vocation INFORMATIVE et, d'autre part, leur texte à caractère RÉFLEXIF.

2 Cinéma 76, no 210 (juin 1976) parle d'«élucubrations oiseuses pour masochistes ay'ant du fric à perdre».

3 Vincent Ostria a rendu compte des trois étapes principales de la mise au point du cinéma holographique: «Les débuts de la cinéholographie», "Cinéholographie, ça continue», "Cinéholographie, de nouveaux progrès", Cahiers du cinéma 340 (octobre 1982), 367 (janvier 1985), 375 (septembre 1985).

4 Depuis, une luxueuse revue de cinéma a pris le même motif pour sa publicité.

5 Voir Jacques Kermabon, «Le cinéma holographique, entretien épistolaire avec Claudine Eizykman et Guy Fihman", "Les Théories du cinéma aujourd'hui», CinémAction 47 (1988) p. 144-150. 


\section{OUVRAGES CITÉS}

Du cinéma selon Vincennes (dirigé par le Département d'études cinématographiques et audio-visuelles de l'Université de Paris VIII). Paris: Lherminier, 1979.

Eizykman, Claudine. La Jouissance-cinéma. Paris: U.G.E., 1976.

Eizykman, Claudine. «Melba: un claquement de rubans, un bruissement de feuilles, un cillement de l'œil». "Cinémas d'avant-garde (expérimental et militant)». CinémAction 10-11 (printemps-été 1980).

Kermabon, Jacques. «Cinquante théoriciens». «Les théories du cinéma aujourd'hui». CinémAction 47 (1988).

Noguez, Dominique. Cinéma: théorie, lectures. Paris: Klincksieck, 1978.

Sudre, Alain. Notice du dictionnaire de Prédal, René. 900 cinéastes français d'aujourd'hui. Paris: Cerf, 1988. 


\section{Dimension du cinéma holographique}

\section{Claudine Eizykman et Guy Fihman}

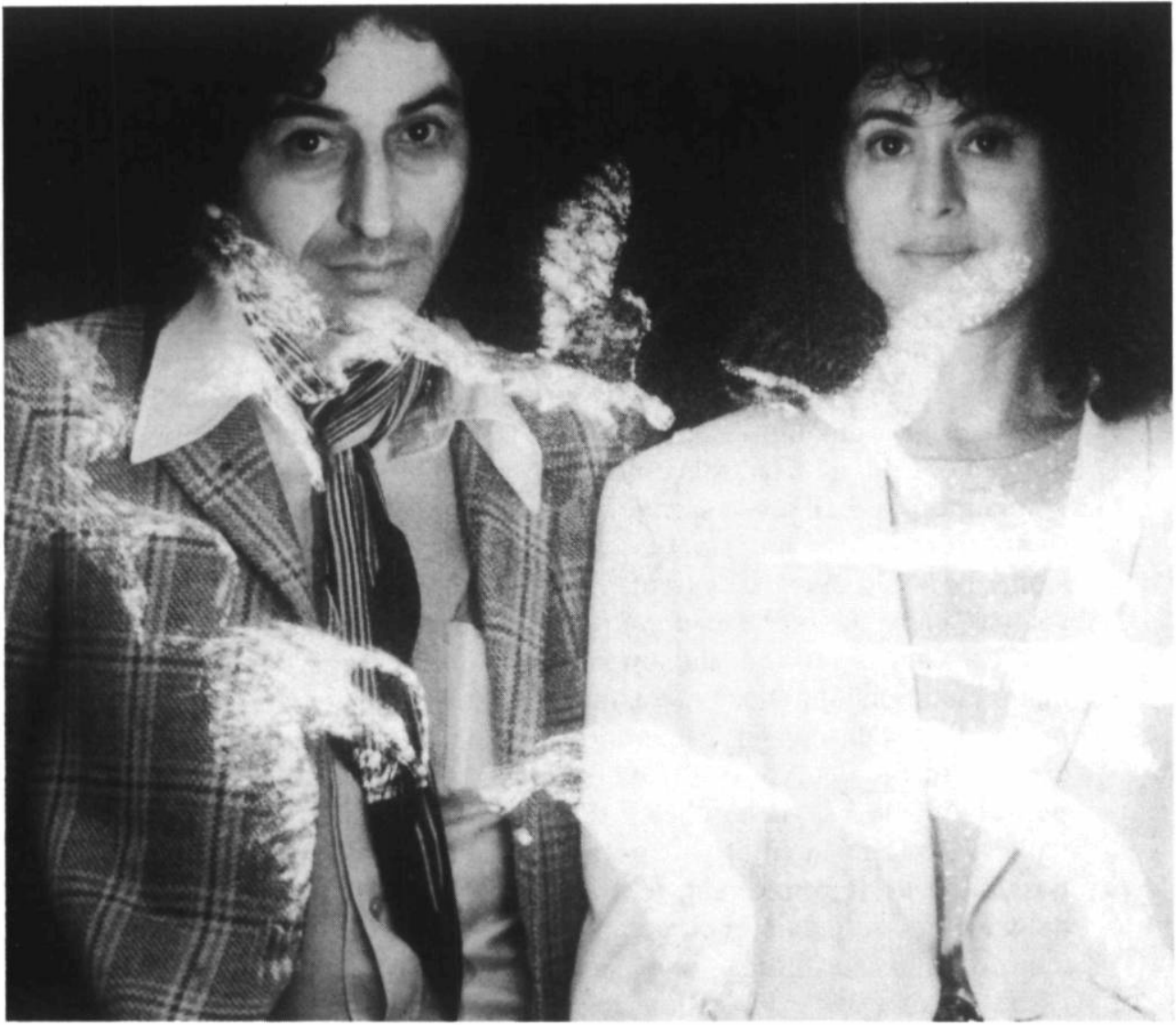

Les Vols d'oiseaux entourant leurs créateurs, $\mathrm{Cl}$. Eizykman et G. Fihman (1982)

Depuis une dizaine d'années les auteurs ont entrepris de concrétiser le cinéma dans sa modalité holographique. La démarche adoptée a consisté à reprendre le processus d'évolution qui a conduit au siècle dernier de la photographie animée au cinéma photographique, en transposant ce processus d'innovation pour le cinéma holographique.

Parmi les nombreuses questions que ce projet et cette démarche suscitent, nous nous proposons d'examiner ici, pour ce numéro de la revue Cinémas consacré à la question «Nouvelles 
technologies: nouveaux cinémas?», plus particulièrement trois aspects: qu'apporte la cinéholographie? pourquoi le relief? quelles relations y a-t-il entre expérimentation artistique et expérimentation technologique? Une réponse lapidaire consisterait à dire que le relief (ajouté au mouvement et à la couleur) répond à une exigence artistique; que l'holographie est seule à même de satisfaire pleinement cette exigence; et de là résulte le projet de cinéma holographique. Mais pour éclairer cet enchaînement de déterminations, il convient de reprendre d'abord la situation des différentes technologies d'imagerie à l'égard de la troisième dimension.

Dans la réalité, la profondeur est la dimension dans laquelle nous évoluons de manière continuelle, et qui donc mérite comme telle d'être considérée comme la première dimension. Ce n'est que dans le domaine de l'image que la profondeur, de première dimension, devient la troisième. Pour bien comprendre ce changement ordinal, il est essentiel de se rappeler que l'image aérienne formée par une optique classique est elle-même tridimensionnelle. L'image ne devient plane que lorsqu'elle est observée sur un écran ou fixée sur un support, toile, tableau ou plan-film.

C'est donc avant tout du support plan que l'image tient sa planéité bidimensionnelle. Devenue alors troisième dimension, la profondeur n'en conserve pas moins sa place éminente. Mais en creux, si l'on peut dire, ou en négatif. C'est parce qu'elle est perdue qu'elle est recherchée. Et c'est parce que la bidimensionalité du support semble a priori exclure la profondeur, que la possibilité de retrouver sur ce même support, non pas la profondeur elle-même, mais quelque chose qui permet de l'évoquer, est tenue depuis au moins cinq siècles pour un prodige, comme l'atteste l'abondante littérature consacrée à la perspective. Il faut même dire que c'est cette présence singulière de profondeur absente qui confère à la perspective son statut de forme symbolique, pour reprendre le titre de l'ouvrage classique de Panofsky. On connaît le mécanisme psychique par lequel la partie retrouvée d'un objet perdu parvient à combler le manque. Il en va de même ici, et la trace de la profondeur perdue sera prise pour sa présence absolue.

Dès lors, en définissant la perspective comme l'art ou la science de représenter sur un tableau les objets visibles comme ils paraissent à la vue, la voie est ouverte pour que se développe le thème de l'illusion. Mais celle-ci tient à une condition, qui reste le plus souvent passée sous silence quand elle n'est pas tout simplement ignorée, au propre ou au figuré. 

Vinci:

Pourquoi la peinture ne peut avoir autant de relief que les choses mêmes? Les peintres désespèrent souvent de ne pouvoir imiter la nature, voyant que leurs œuvres n'ont pas ce relief et cette réalité qu'ont les choses vues dans le miroir, bien qu'ils disposent, disent-ils, de couleurs beaucoup plus claires ou plus foncées que les tons des ombres et des lumières de l'objet vu dans un miroir; et ils accusent de cela leur ignorance, et non la vraie raison, qu'ils ne connaissent pas: c'est qu'il est en effet impossible que la chose peinte apparaisse avec autant de relief que la chose vue dans un miroir, bien que l'un et l'autre se trouvent dans un plat, - à moins qu'on ne les regarde avec un seul oeil.

Le rendu perspectiviste de la profondeur exige du peintre comme du regardeur cette condition monoculaire. Un seul œil immobile, cette condition se retrouve dans toutes les formes d'imagerie - telle que la photographie - , qui ont la perspective pour fondement et qui, en fait d'art visuel, devraient être tenues pour le stade borgne de l'art visuel.

Contemporaine de la photographie, la stéréoscopie a semblé être le moyen de surmonter cette limitation monoculaire de l'image: la stéréoscopie n'étant que l'accomplissement binoculaire de la perspective, une vue pour chaque oeil, pouvait se combiner avec les perspectives daguériennes. La stéréophotographie était alors censée reproduire le relief de la vision naturelle. Il n'est pas rare de retrouver aujourd'hui encore cette affirmation, même si l'on sait que ce n'est pas le cas: la stéréoperspective ne comporte qu'une dimension de parallaxe et ne permet pas de reproduire à la fois toutes les qualités de reliefs (texture, modelé, volume, profondeur); en outre, elle impose à la vision une rupture de la synergie accommodation-convergence. Mais qu'importe, la stéréo en rendant la vision des objets quasiment solide, retrouvait une autre partie perdue de la profondeur et, du coup, faisait voir comme plates les simples perspectives picturales ou même photographiques, que dans le jargon des photographes du XIXe siècle on appelait «mono». Une ou deux photographies, ces vues restaient cependant fixes, impliquaient toujours une position unique d'enregistrement et d'observation.

Après avoir connu une vogue fantastique, la stéréophotographie est redevenue une technique ou une pratique spécialisée. Mais il y a beaucoup plus qu'une simple coïncidence dans le déclin de la stéréo qui s'accompagne historiquement du développement du cinéma. Car la série des vues photographiques n'apportait pas seulement le mouvement. Par l'écart de positions des objets, mais encore plus par les écarts de points de vue dès qu'il y a mouvement de la caméra, une certaine profondeur était 
comme miraculeusement restituée à partir des images plates et fixes. Tout se passe comme si un seul oeil susceptible de faire varier ses positions faisait office de l'organe complet de la vision. Le mouvement comble cette profondeur atténuée, en la rendant beaucoup plus prégnante que celle des simples photographies. Si l'effet ainsi produit n'est plus considéré comme la reproduction de la réalité, «la vie même» comme disaient les contemporains du Cinématographe, les théoriciens du cinéma qui maintiennent la fameuse impression de réalité ne font guère que dire la même chose autrement. Quant à la profondeur, que font d'autre les spécialistes de l'image de synthèse lorsqu'ils évoquent, comme le nec plus ultra de leur technique, l'animation en «3 D»? Dans ce cas la modélisation mathématique est certes tridimensionnelle, mais pour aboutir à une série d'images bidimensionnelles, c'est-à-dire pas plus et plutôt moins que la série d'images analogiques qui est au principe du cinéma: un seul point de vue qui peut se déplacer dans un espace dans lequel des personnages et des objets évoluent. Et si un plus de profondeur et de relief est visuellement requis, l'imagerie de synthèse n'a d'autre recours que la voie explorée par le cinéma depuis ses origines, qui est de s'adjoindre le relief stérêoscopique. Â cet égard, il en va exactement de même et ceci n'est pas indifférent pour les procédés Imax et même Omnimax.

Si toutes ces techniques sont à la recherche de la profondeur perdue, c'est que leur fondement commun reste la perspective, que les images soient graphiques, photographiques ou électroniques, analogiques, synthétiques ou hybrides.

Avant même le relief qui est son effet principal, l'importance essentielle de l'holographie réside dans le fait que, par principe, l'image holographique ne procède pas de la perspective. D'où la difficulté à l'appréhender conceptuellement, et corollairement son importance théorique cruciale pour le renouvellement des concepts constitutifs de l'image.

En effet, une perspective se caractérise par une relation strictement biunivoque entre la scène tridimensionnelle et l'image bidimensionnelle: à un point de l'une correspond un point et un seul de l'autre, et réciproquement. L'holographie, au contraire, se caractérise par une relation totalement multivoque: chaque partie de l'hologramme contient la totalité de la scène enregistrée. La portée de cette différence est considérable. Elle exclut que l'on puisse manuellement faire un hologramme; elle interdit que l'on puisse parler d'un «pixel» holographique: en effet un élément d'image holographique est déjà un hologramme, qui ne peut se réduire à un point mais doit occuper plus que la surface, 
un certain volume dans l'épaisseur de l'émulsion. Ceci résulte comme on le sait de l'inscription interférentielle de l'information, qui prend en compte la phase et l'amplitude de la lumière cohérente, là où l'imagerie traditionnelle, c'est-à-dire perspectiviste, ne tient compte que de l'intensité de la lumière incohérente.

Le résultat visuel de cette modalité est que l'hologramme se comporte comme une fenêtre, qui n'est plus limitée à un seul point de vue comme la fenêtre perspectiviste, mais qui présente tous les points de vue qu'offrirait une fenêtre réelle: l'objet ou la scène sont directement en relief et situés dans l'espace.

De par sa modalité d'inscription interférentielle de l'information, l'holographie présente, par rapport à l'imagerie perspectiviste traditionnelle, un certain nombre de particularités qui, à l'analyse, se révèlent être des déterminations strictes des constituants internes de l'image. Ceci résulte du fait que pour l'holographie la lumière ne joue pas seulement sa fonction traditionnelle d'éclairage de la scène, mais intervient simultanément dans le principe même d'enregistrement. Ainsi la classique profondeur de champ se transforme en volume de cohérence, qui dépend essentiellement des paramètres du laser utilisé et de la configuration du montage optique d'enregistrement: n'est enregistré que ce qui est éclairé de manière cohérente.

Si l'holographie présente les objets ou la scène en relief, il ne s'agit cependant pas, à bien des égards, d'une stricte reproduction de la réalité telle qu'elle s'offre à notre vision, ne serait-ce que par l'impossibilité, sauf à utiliser des subterfuges, d'enregistrer un vaste panorama ou une source de lumière naturelle ou artificielle. Ici ressurgit le problème de la «mimesis», qui a presque toujours été recouvert par l'a priori du réalisme.

Gabriel Lippmann, inventeur du procédé de photographie interférentielle des couleurs dont on célèbre cette année le centenaire, faisait remarquer en commentant l'expérience de base qui consiste à projeter sur un écran l'image des objets éclairés, que «l'image paraît toujours jolie, alors même que l'objet nous est indifférent» et il ajoutait que «le plaisir qu'on trouve à la regarder a peut-être ses raisons en dehors de la physique». D'où vient ce plaisir, cet intérêt voire cette fascination que l'image, toute image, suscite? Les tenants du réalisme s'empressent d'affirmer que l'image tient son statut du fait qu'elle est image de quelque chose du monde, du réel. Mais alors comment expliquer cet intérêt pour l'image alors même que l'objet est indifférent? C'est que même dans ce cas l'image nous concerne, elle est de notre monde, ou pour le dire autrement, c'est notre perception visuelle qui appartient au monde de l'image. C'est ce qu'indiquent les 
peintres au moins depuis l'impressionnisme, lorsqu'ils affirment ne pas peindre ce qui est mais ce qu'ils voient. Cette attitude, qui s'oppose au réalisme, pourrait dans sa généralité être désignée sous le nom de visualisme, en ce qu'elle accorde la primauté à la vision sur le monde vu.

Les auteurs se rangent parmi les tenants du visualisme, ainsi entendu. Et, de manière plus précise, c'est l'exigence esthétique (résultant de nos travaux préalables en cinéma expérimental) d'un art visuel pleinement abouti en ce qu'il comporterait les qualités perceptives visuelles essentielles que sont le mouvement, le relief intégral et les couleurs véritables (spectrales et non plus pigmentaires), qui nous a conduit au projet de concrétisation du cinéma holographique, seule modalité susceptible de présenter ces qualités sans lesquelles un art ne peut être pleinement visuel.

Le changement de technologie, du cinéma photographique ou électronique au cinéma holographique, n'implique pas un changement du but poursuivi. Bien au contraire, puisque c'est celui-ci qui détermine celui-là. L'expérimentation technologique ne s'oppose donc pas à l'expérimentation artistique; elle en est le prolongement direct, la conséquence cohérente. Pour les visualistes convaincus que nous sommes, le cinéma holographique est un enjeu trop important pour être laissé aux techniciens, comme le voudrait une division du travail ininterrogée. L'intervention des artistes sur les moyens de leur art fait partie d'une longue tradition et il est étonnant que cela puisse susciter l'étonnement.

Université de Paris VIII 